\title{
Refractory periods and climate forcing in cholera dynamics
}

\author{
Katia Koelle ${ }^{1 \star}$, Xavier Rodó $^{2 \star}$, Mercedes Pascual $^{1}$, Md. Yunus $^{3} \&$ Golam Mostafa $^{3}$
}

Outbreaks of many infectious diseases, including cholera, malaria and dengue, vary over characteristic periods longer than 1 year ${ }^{1,2}$. Evidence that climate variability drives these interannual cycles has been highly controversial, chiefly because it is difficult to isolate the contribution of environmental forcing while taking into account nonlinear epidemiological dynamics generated by mechanisms such as host immunity ${ }^{2-4}$. Here we show that a critical interplay of environmental forcing, specifically climate variability, and temporary immunity explains the interannual disease cycles present in a four-decade cholera time series from Matlab, Bangladesh. We reconstruct the transmission rate, the key epidemiological parameter affected by extrinsic forcing, over time for the predominant strain (El Tor) with a nonlinear population model that permits a contributing effect of intrinsic immunity. Transmission shows clear interannual variability with a strong correspondence to climate patterns at long periods (over 7 years, for monsoon rains and Brahmaputra river discharge) and at shorter periods (under 7 years, for flood extent in Bangladesh, sea surface temperatures in the Bay of Bengal and the El NiñoSouthern Oscillation). The importance of the interplay between extrinsic and intrinsic factors in determining disease dynamics is illustrated during refractory periods, when population susceptibility levels are low as the result of immunity and the size of cholera outbreaks only weakly reflects climate forcing.

Host immunity has a key role in the nonlinear dynamics of infectious diseases. Acquired immunity and the replenishment of susceptible individuals are capable of generating interannual disease cycles in nonlinear mathematical models of disease lacking any environmental forcing ${ }^{5}$. Seasonality can interact with this natural frequency to produce oscillations of longer period or more complex patterns, including chaos ${ }^{6}$. Thus, interannual disease cycles can either arise intrinsically, by means of epidemiological dynamics including seasonality, or be driven extrinsically, for instance by interannual climate variability at frequencies similar to those of the response in incidence levels. No evidence has yet been obtained for a role of climate variability in interannual disease cycles with an approach that allows for the alternative explanation that they are simply produced intrinsically ${ }^{4}$. The few exceptions have so far been limited to phenomenological treatments of disease dynamics ${ }^{7,8}$. We use a nonlinear population model that takes into account immunity and disease transmission to show a strong correspondence between cholera transmission and climate variability.

The temporal cholera data consist of monthly symptomatic cases from the rural region of Matlab, Bangladesh, from 1966 to 2002, obtained from a surveillance programme by the International Center for Diarrhoeal Disease Research (Fig. 1). The study area is located $40 \mathrm{~km}$ southeast of the capital Dhaka, and lies in the delta region of the Ganges and the Brahmaputra rivers. We focus the analysis on the temporal variability of the predominant strain, El Tor, whose dynamics exhibit clear seasonal and interannual variability, with peaks in spring and late autumn'.

To separate the roles of intrinsic feedbacks from extrinsic (environmental) forcing, we extended a recently proposed disease model ${ }^{10}$ to incorporate both immunity from previous El Tor infections and the likely possibility of cross-immunity from previous infections caused by the Classical strain (see Methods). The model contains two equations. The first one is a nonlinear transmission equation of the form

$$
I_{t+1}=\beta_{t} I_{t}^{\alpha}\left(\frac{S_{t}}{N_{t}}\right)^{\gamma} \varepsilon_{t}
$$

where $I_{t}$ is the number of El Tor-infected individuals, $S_{t}$ is the number of individuals susceptible to El Tor, $N_{t}$ is the total population size, and $\varepsilon_{t}$ is a multiplicative noise term, all at time $t$. The exponents $\alpha$ and $\gamma$ are used to incorporate deviations from the random mixing assumption $^{11}$. Pathogen transmission rate $\beta_{t}$ is a key parameter that we specifically let vary in time, to represent the effect of extrinsic
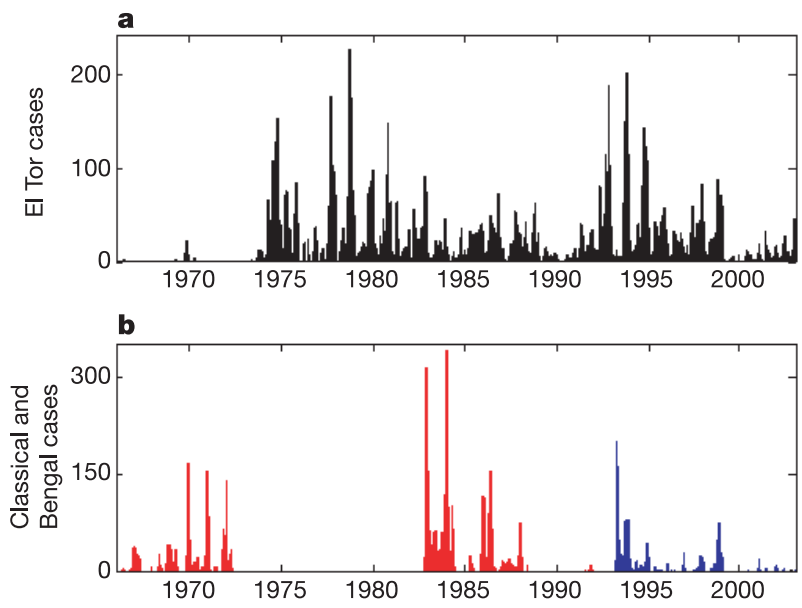

Figure 1 | Time series of cholera cases from 1966 to 2002, aggregated monthly. a, Cholera cases of the El Tor biotype. b, Cholera cases of the Classical biotype (red), and the Bengal strain (blue). Bengal cases were excluded before analysis because this strain belongs to a different serogroup (0139) from El Tor and Classical (01), and cross-immunity between serogroups seems to be absent ${ }^{28}$. Population size was estimated using four census points and monthly demographic data adjusted for net emigration, and increased from 152,000 in 1966 to 223,000 in 2002 (not shown). 
drivers on the transmission rate. This parameter corresponds to the number of contacts per infected individual per unit time, multiplied by the probability that a contact with an infected individual leads to infection $^{12}$. For cholera, contacts reflect faecal-oral transmission through food and water, and/or environmental transmission through contamination of aquatic environments used by humans. $\beta_{t}$ contains both a seasonal component $\beta_{\text {seas }}$ and a longer-term component $\beta_{\mathrm{lt}}$, such that $\beta_{t}=\beta_{\text {seas }} \beta_{\text {lt }}$ (ref. 10). We divide $\beta_{t}$ into two components to identify fluctuations in transmission rates that seasonality alone cannot explain. In particular, this formulation allows for mechanisms that influence interannual variability in $\beta_{t}$ as the result of a modulation of the seasonality in transmission rates. A second equation specifies the number of susceptible individuals at time $t$ :

$$
S_{t}=N_{t}-\sum_{i=0}^{m}\left(\kappa_{i} I_{t-i}\right)-\sum_{i=0}^{m}\left(\kappa_{i}^{\mathrm{cl}} I_{t-i}^{\mathrm{cl}}\right)
$$

where $N_{t}$ is the current population size at time $t, \sum_{i=0}^{m}\left(\kappa_{i} I_{t-i}\right)$ is the total number of individuals recovered from an El Tor infection and immune to El Tor reinfection, and $\sum_{i=0}^{m}\left(\kappa_{i}^{\mathrm{cl}} I_{t-i}^{\mathrm{cl}}\right)$ is the total number of individuals recovered from infection by the Classical biotype and immune to El Tor reinfection. The functions $\kappa_{i}$ and $\kappa_{i}^{\mathrm{cl}}$ describe the decay of immunity, with the subscript $i$ indicating the number of
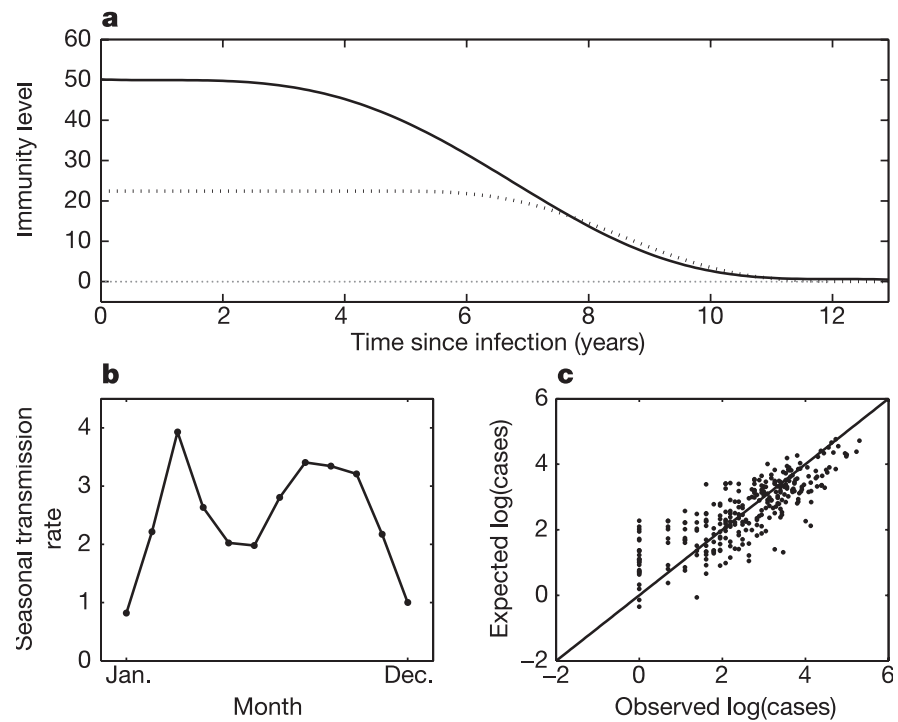

Figure $2 \mid$ Results of the nonlinear disease model. a, The decay of immunity functions $\kappa_{i}$ and $\kappa_{i}^{\mathrm{cl}}$ for individuals having recovered from previous El Tor (solid line) and Classical (dotted line) infections, respectively. The decay of immunity quantifies the duration for which previously infected individuals are expected to be immune to reinfection with cholera of the El Tor biotype. The intercepts $\kappa_{i}$ and $\kappa_{i}^{\mathrm{cl}}$ correspond to the ratio of asymptomatic to symptomatic infections ${ }^{10}$. This assumes that immunity is initially complete, a reasonable assumption given results from rechallenge studies ${ }^{14}$. The infection-to-case ratios estimated from these intercept values correspond to 50:1 for El Tor and 22:1 for Classical. These results fall within the published range of asymptomatic to symptomatic infection ratios for cholera ${ }^{29}$ and are in agreement with the known higher asymptomatic ratio for El Tor than Classical $^{30} \cdot \mathbf{b}$, The seasonal component of the transmission rate, $\beta_{\text {seas }}$, anchored at its normalized December value $\beta_{\mathrm{dec}}$, so that $\beta_{\mathrm{dec}}=1$. c, Logarithm of expected El Tor cases plotted against the logarithm of observed El Tor cases $\left(r^{2}=0.63\right)$. The mixing exponent $\alpha$ equals 0.57 with $\gamma$ set at 1 (Supplementary Information). The low value of $\alpha$ might reflect cholera's high degree of spatial clustering, a pattern noted empirically ${ }^{9}$. Alternatively, because $V$. cholerae temporarily survives in an aquatic reservoir, the dependence on the previous month's infections might be lower than for diseases with a strict direct transmission route. Dynamic simulations of the model without noise generate strict annual cycles. When simulated with dynamic noise, interannual variability does arise, but no regularities in dominant frequencies are found. months since infection and the values of $\kappa_{i}$ and $\kappa_{i}^{\mathrm{cl}}$ being the degrees of immunity that an individual has $i$ months after being infected. Equations (1) and (2) are combined into a single expression relating incidence levels in the present to those in the past. The fit of this model to data relies on a semi-parametric approach ${ }^{10,13}$ because the long-term component of the transmission rate $\left(\beta_{1 \mathrm{t}}\right)$, which incorporates any trends or fluctuations of periodicity longer than seasonal, is not specified or constrained in any way. The model itself reconstructs the patterns of time variation in transmission and associated susceptible levels. It further provides an estimate of the immunity and cross-immunity functions. Acquired immunity to Vibrio cholerae is known to exist, but its duration is highly debated ${ }^{14}$.

Results show that acquired immunity to reinfection with El Tor, from previous Classical and El Tor infections, is long-lasting (Fig. 2a). The degree of immunity from a previous El Tor infection starts to wane 3 years after infection, but partial immunity lasts for up to 10 years. Classical infections confer complete cross-immunity for more than 6 years, and full susceptibility to El Tor reinfection does not occur until more than 10 years after infection. A shorter duration of El Tor immunity than Classical cross-immunity agrees with results from field studies ${ }^{15}$, and may result from the greater severity of Classical infections producing stronger immunological responses. The intercepts of the immunity functions can be interpreted as providing estimates of ratios of asymptomatic to symptomatic cases (Fig. 2a). The higher ratio for El Tor than for Classical infection agrees with previous epidemiological studies (Fig. 2, legend). Seasonal transmission shows a clear monthly variation (Fig. 2b) consistent with the known seasonality of cases. More significantly, from the immunity curves, the fraction of the population susceptible to El Tor infection (the ratio of susceptible to total individuals; $\mathrm{S} / \mathrm{N}$ ) over time can be computed from equation (2) (Fig. 3b). Concurrent changes in the long-term transmission rate (Fig. 3a) provide evidence for the forcing of cholera by extrinsic factors at interannual timescales. The fit of the full model accounts for $63 \%$ of the variability in the logarithm of the El Tor cases (Fig. 2c). Additional effects of interannual forcing might still be contained in the residuals of the model, a point to which we return later when addressing specific environmental covariates.

We can now look more closely at the distinct roles of extrinsic (environmental) drivers and intrinsic (immunity) factors in the dynamics of cholera. Figure 3 shows the long-term transmission rate temporally aligned with the fraction of the host population susceptible to El Tor infection, as well as the cholera case data aggregated monthly. In times of high transmission, the response in El Tor cases would be expected to be large if intrinsic factors were
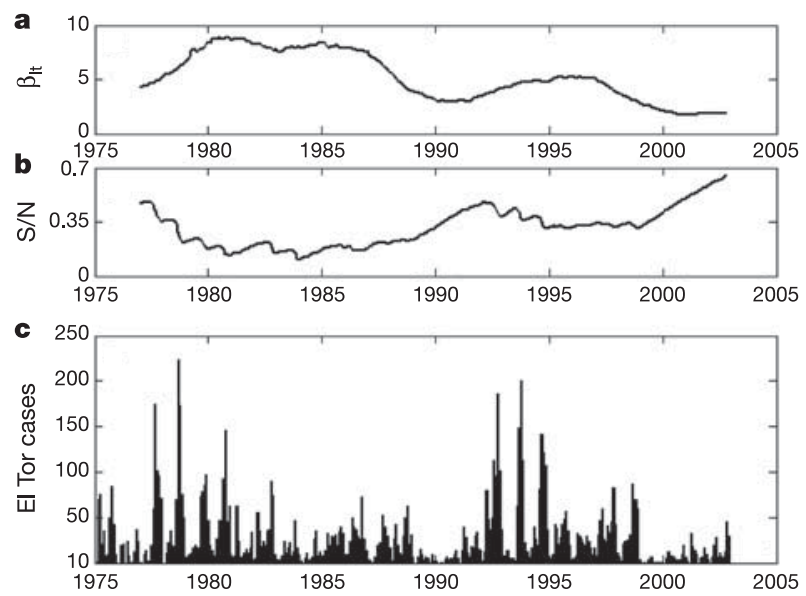

Figure 3 | Cholera refractory periods. a, The long-term component of the transmission rate, $\beta_{1 \mathrm{t}} . \mathbf{b}$, The fraction of the population susceptible to cholera (S/N) over time. c, Time series of El Tor cases from 1976 to 2002. 
unimportant. However, the magnitude of the response is only high at a subset of these times. Most notably, El Tor cholera cases are low for 1982-87, despite the favourable environmental conditions for high transmission of pathogens. This episode was concurrent with a low proportion of susceptible hosts after the higher incidence levels of the late 1970s and early 1980s (including the large Classical outbreak in 1982-83). Moreover, one of the highest El Tor cholera case episodes (1993-95) occurred when transmission rate was only moderately high but the susceptible fraction had had significant time to replenish itself (1988-92). These results illustrate that high transmission rates result in high case responses only when the immunity levels of the host population are low; that is, when the host population is not in a refractory period from previous disease outbreaks.

We now turn to the interpretation of changes in interannual patterns of transmission by examining associations with potential climatic drivers. These associations provide support for the model itself and for the assumption that the reconstructed variability in transmission reflects extrinsic forcing. We focus on rainfall, associated river discharge, and flood extent, because of the prominent role of water levels in the proposed mechanisms for cholera seasonality ${ }^{16}$. In the bimodal seasonal cycle of cholera cases (and also of transmission rates; Fig. 2b) there is a marked decrease during the summer monsoons, probably resulting from a reduction in cholera's environmental concentration and/or the decrease in salinity affecting its survival. Cholera cases increase again and peak with a lag after this season, as floods presumably concentrate the population on the decreased land area available and break down sanitary conditions, promoting secondary transmission through the more direct faecaloral route. These two seasonal mechanisms of opposite effect at different lags can therefore mediate an influence of either positive or negative rainfall anomalies at interannual timescales. Besides rainfall, we also consider two remote drivers of interannual climate variability in the region, the El Niño-Southern Oscillation (ENSO) and sea surface temperatures (SSTs) in the Bay of Bengal, previously proposed to influence cholera in Bangladesh ${ }^{8,17}$. Figure 4 a shows a clear inverse relationship between the reconstructed transmission rate $\beta_{\text {lt }}$ and both the low-frequency variation of rainfall from northeast India ${ }^{18}$ and Brahmaputra river discharge data, providing the first evidence for the long-standing hypothesis that rainfall and associated water levels drive cholera patterns. A long-term decreasing trend in transmission rate, associated with an opposite long-term trend in the Brahmaputra's discharge, is also evident. These environmental time series components were obtained by separating signal from noise and isolating the variability with a period longer than 7 years (see Methods). This period was chosen to focus initially on interannual variability at scales longer than those relevant for ENSO.

A second curve is shown in Fig. 4a for the long-term component of the transmission rate that closely matches the estimated $\beta_{\mathrm{lt}}$. This curve was obtained by first aggregating the logarithm of the fitted long-term transmission rate, $\log \beta_{\mathrm{lt}}$, with the residuals from the model, $\log \varepsilon_{t}$. This non-seasonal transmission term allows us to consider all possible influences on incidence levels that were not accounted for by the epidemiological dynamics and seasonal forcing. The low-frequency component of transmission was then isolated from this aggregated variable, by using the same procedure as for the environmental variables. This approach produces a curve extremely similar to that originally fitted by the model. Interestingly, additional frequency components of this aggregated variable can now be isolated at shorter timescales, particularly those relevant to ENSO. Figure $4 \mathrm{~b}$ shows the signal extracted for variability at periods less than 7 years superimposed on the SST anomalies averaged for the Niño3.4 region in the Pacific, and SST anomalies averaged for the Bay of Bengal. The SST in the Bay of Bengal lags the SST anomalies for the Niño3.4 region (Fig. 4b) by $2-3$ months, establishing a connection between ENSO events and regional climate in Bangladesh, which we discuss below. In particular, there is a strong positive lagged correlation of this faster component of transmission with the 1987-88 and the 1997-98 El Niño events. Locally, extreme floods in Bangladesh (Fig. 4b) and positive rainfall anomalies (Supplementary Fig. S3) occur during these ENSO years. A positive association between SST in the Bay of Bengal and this component of transmission is also evident. The 1987-88 ENSO episode is of special relevance because, despite the increase in transmission rate, the response in terms of cases occurs but is a weak one (Fig. 3c). This episode falls during the refractory period, for which the susceptible population is small and not yet ready to respond to increases in transmission rate. Only the
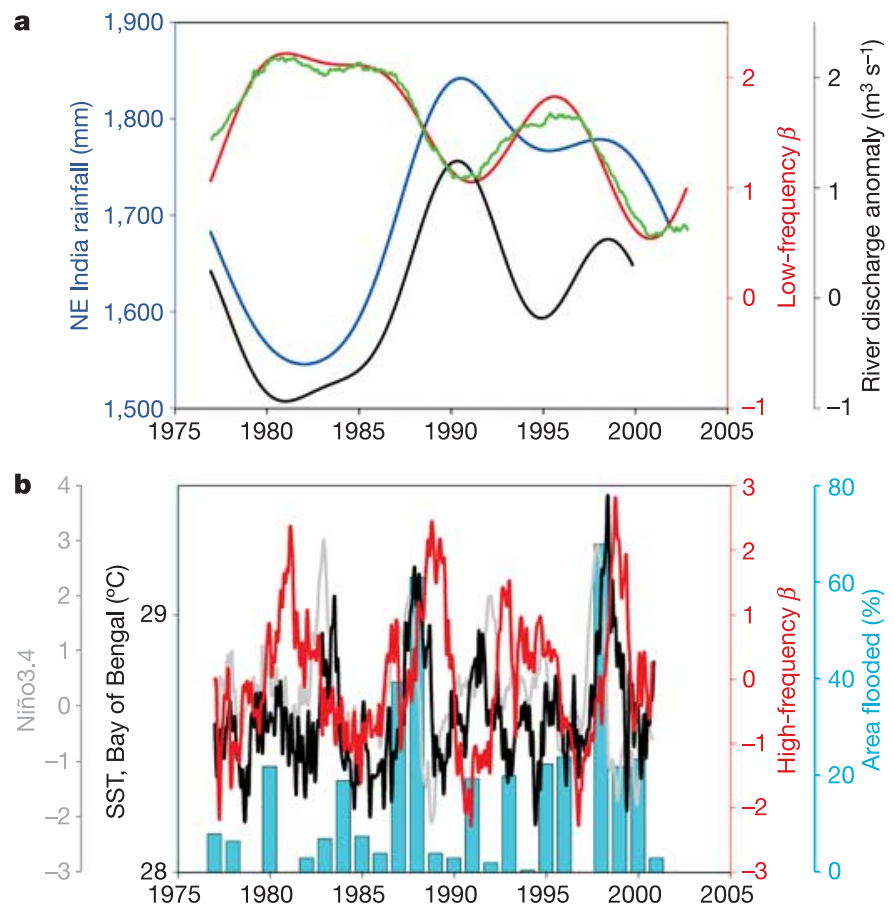

Figure 4 | Environmental drivers and transmission. The non-seasonal component of the transmission rate (green; plotted as $\log \beta_{1 t}$ ) was added to the residuals of the model, and decomposed into frequencies of low and high variability (accounting for $27 \%$ and $57 \%$, respectively, of the variance in this aggregated term). a, The low-frequency variation in the non-seasonal transmission rate (red), plotted with the low-frequency variation of northeast (NE) India rainfall (blue) and Brahmaputra river discharge (black). NE India rainfall covers an area of four subdivisions and $267 \times 10^{3} \mathrm{~km}^{2}$. As expected, the low-frequency variation of the NE India rainfall is reflected in the Brahmaputra river discharge variation $(r=+0.93$, $P<0.05$, lag $=0$ months). The low-frequency variation in transmission is negatively correlated with both the NE India rainfall $(r=-0.797, P<0.05$, lag $=14$ months) and Brahmaputra river discharge anomalies

( $r=-0.9278, P<0.02$, lag $=7$ months). $\mathbf{b}$, The high-frequency variation in the non-seasonal transmission rate (red), plotted with Niño3.4 (grey) and SST in the Bay of Bengal (black). SSTs in the Bay of Bengal are obtained from a $0^{\circ}$ to $23^{\circ} \mathrm{N}$ and $80^{\circ}$ to $100^{\circ} \mathrm{E}$ grid, with $2^{\circ} \times 2^{\circ}$ resolution from the extended reconstructed SSTs of the National Oceanic and Atmospheric Administration National Climate Data Center. Local correlations between Niño3.4 and SST in the Bay of Bengal reach +0.86 , with a 2-3-month lag $(P<0.05)$. Local correlations between the high-frequency component of transmission and Niño3.4 reach maximal values between +0.63 and +0.91 at 8-10-month lags $(P<0.05)$ for 1986-87, 1990-91, 1994 and 1997-98. Similarly, local correlations between this transmission component and SST in the Bay of Bengal range between +0.68 and +0.96 for $0-9$-month lags $(P<0.05)$ for 1982, 1986-87, 1988-89, 1990-91 and 1997. Bars show the percentage of the area of Bangladesh flooded over this time period. Annual flood area data come from the flood forecasting and warning centre (BWDB, Dhaka, Bangladesh). An area is considered flooded if it experiences at least one flooding event within the year's monsoon season. Local correlations between SST in the Bay of Bengal and NEIR (northeast India rainfall) (not shown) are negative, exceeding -0.85 at interannual timescales for periods longer than 7 years $(P<0.0001)$. 
episode of 1982-83, for which the response is predominantly Classical and not El Tor, shows no increase in transmission rate. A time-series method that specifically quantifies the strength of the association between variables locally in time (see Methods) reveals that only one particular biotype at a time is responsible for the response of cases to climate for a given El Niño event (Supplementary Fig. S1).

The comparison of regional rainfall anomalies for Bangladesh between the different ENSO events reveals that 1982-83 exhibited drought conditions that were unique for this period in their intensity and spatial extent (Supplementary Figs S2 and S3). This drought provides an explanation for the major outbreak of Classical infection in place of El Tor. Anomalously low water levels (and associated higher salinity) have been proposed to favour this biotype relative to El Tor in spatial observations of biotype distributions in Bangladesh, with Classical found in more coastal regions ${ }^{19}$.

Thus, both floods and droughts seem to promote cholera transmission, depending on the strain and the temporal scale of interannual variability. This finding is consistent with the two different ways in which water levels have been postulated to influence the seasonality of cholera. This complex nonlinear response to water levels should be examined further with mechanistic mathematical models that couple cholera seasonality to its interannual variation. Our findings on SST in the Bay of Bengal (Fig. 4b) further support a role of rainfall and clarify the regional influence of climate variability on cholera at ENSO timescales. Two areas for SST in the Indian Ocean appear most relevant to the variability in Bangladesh rainfall, namely the central Indian Ocean ${ }^{20}$ and the Bay of Bengal. SSTs in these regions exert a (nonlinear) influence on the subsequent summer monsoon $^{21}$. These areas also appear strongly linked to El $\mathrm{Niño}^{22}$ (Fig. 4b for Bay of Bengal), although the degree of their dependency, and that of the so-called Indian Ocean dipole mode, on ENSO remain controversial ${ }^{23}$. In addition to rainfall, water temperature in ponds and rivers provides another local mechanism for the remote association of cholera transmission with SST in the Bay of Bengal and the Pacific (ENSO). Changes in cloud cover, wind stress and evaporation modulate variations in the net heat flux entering the system $^{22,24}$, increasing both the SST in the Bay of Bengal and affecting the surface temperature over land. The resulting warming of water temperature in ponds and rivers might increase the incidence of cholera through the faster growth rate of the pathogen in aquatic environments.

We have shown the existence of refractory periods during which climate-driven increases in transmission do not result in large outbreaks. Once the interplay of climate forcing and disease dynamics is taken into account, clear evidence emerges for a role of climate variability in the transmission of cholera. A nonlinear population model achieves this by explicitly taking into account epidemiological dynamics: changes in the abundance of susceptible hosts, rates of decay of immunity and seasonal transmission could all be reconstructed from time series of infected individuals and total population. The finding of a high susceptible fraction in the Matlab population for recent times is of particular concern for the near future. Although a prolonged period of low transmission rate has been present since 2000, the system seems ripe for an outbreak if this rate were to increase. Future work should explicitly compare the predictability of models based solely on climate variables with that of models including intrinsic disease dynamics ${ }^{8,25}$. Our results suggest that forecasting schemes will require the consideration of both climate variability and the fraction of susceptible individuals in the population. Given that time series on susceptible levels are rarely available, approaches such as that presented here are crucial in the attempt to forecast and anticipate the future size of outbreaks. Realtime monitoring on the state of the oceanic regions we have outlined will also be important for an effective early warning system based on climate, but will need to be integrated with susceptibility levels in future work.

\section{METHODS}

Fitting the extended population model. The extended model seeks to recover the seasonal transmission rates, the long-term transmission rates, the mixing exponent $\alpha$ and the decay of immunity functions $\kappa$ and $\kappa^{\mathrm{cl}}$, given only time series of cholera cases and population size. As in the original nonlinear population model $^{10}$, the transmission equation is logarithmically transformed, making it a semi-parametric additive model. Using a backfitting algorithm, the parametric part of the model is first fitted through a combination of weighted least-squares regressions and recursive Taylor expansions. The backfitting algorithm is necessary to provide a progressive improvement in our estimate of the susceptible fraction in the population through the adjustment of the immunity functions. The nonparametric part of the model-that is, the long-term transmission rate-is then obtained by smoothing the residuals of the regression step. Two parameters (smoothing bandwidth $h$ and spline penalty weight $\mu$ ) determine the flexibility of the model and are objectively selected by crossvalidation (see also Supplementary Information). Reported results shown in Figs 2 and 3 have $h=23$ and $\mu=10^{10}$. The extension of the model to two strains resides in the regression step, where the number of susceptible individuals is expressed as equation (2) for the two-strain model and $S_{t}=N_{t}-\sum_{i=0}^{m}\left(\kappa_{i} I_{t-i}\right)$ for the one-strain model. Further details on this method are described in Supplementary Information.

Issues pertinent to the cholera data set. Case data include all Classical and $\mathrm{El}$ Tor cholera patients from the Matlab surveillance area. After 1978, this area consisted of a maternal, child health and family planning treatment area and a comparison area. Cholera treatment and dynamics did not differ between these areas; we therefore aggregated these two areas in our analyses. Over the period 1966-2003, four vaccine trials were conducted in the Matlab area. To determine whether these trials affected the results, the time series were adjusted (with supplemented cases) to eliminate the protective effect of the vaccines. A fit of the model to these adjusted time series showed no appreciable difference in results (not shown). Implicit in the fit of this nonlinear disease model is the assumption that the generation time of the disease is about 1 month. Although individuals are usually symptomatic for less than a month, infected individuals can shed the bacterium for up to 3 weeks. Further analyses using data aggregated twice a month generate similar results.

Isolation of frequency components from transmission rates and climate data. Eigendecomposition analysis was applied to the climate time series. Eigendecomposition partitions signals on the basis of adaptive nonparametric functions ${ }^{26}$. This technique avoids the bias towards the concentration of noise in certain frequency components. A covariance matrix is first constructed whose entry in row $i$, column $j$ is the covariance of the data at lag $i-j$. The order of the eigendecomposition (that is, the embedding dimension) corresponds to the number of rows in the matrix. Singular value decomposition is then applied to this matrix to extract the eigenvectors and their corresponding eigenvalues. The eigenvalues quantify the variance associated with each eigenvector; they were normalized to have their relative contributions rescaled to $100 \%$. We applied a decomposition of 40th order, which provided a good signal-to-noise separation, and verified that results were insensitive to parameter modifications. Projection of the signal onto the eigenmodes gives the principal components. To reconstruct the time series, the principal components associated with the first group of significant eigenvalues are combined. Six eigenmodes were kept here because they maximized signal-to-noise ratios. Residuals passed all white noise tests. The resulting reconstructed component was filtered with wavelet analysis to separate the variance into two components, one with periods more than 7 years and the other with periods between 1 and 7 years.

Global and local correlation coefficients. To determine the significance of global linear correlations between two time series that share a similar range of preselected periods, we used a bootstrap method that generates 10,000 surrogate data sets for one of the time series by randomizing the phases while preserving the power spectrum and autocorrelation function ${ }^{27}$. Previous work has shown that at ENSO timescales, associations between cholera and climate variability can be discontinuous in time ${ }^{17}$. We therefore also used a time series method (scale-dependent correlation analysis) that specifically quantifies the strength of the association between variables locally in time by using correlation coefficients computed within short, truncated time windows. See the legend to Supplementary Fig. S1 for further details and an example.

\section{Received 1 April; accepted 16 May 2005}

1. Bouma, M. J. \& Pascual, M. Seasonal and interannual cycles of endemic cholera in Bengal 1891-1940 in relation to climate and geography. Hydrobiologia 460, 147-156 (2001)

2. Hay, S. I. et al. Etiology of interepidemic periods of mosquito-borne disease Proc. Natl Acad. Sci. USA 97, 9335-9339 (2000)

3. Taubes, G. Global warming: Apocalypse Not. Science 278, 1004-1006 (1997) 
4. Rogers, D. J. et al. Satellite imagery in the study and forecast of malaria. Nature 415, 710-715 (2002)

5. Anderson, R. M. \& May, R. M. Infectious Diseases of Humans: Dynamics and Control (Oxford Univ. Press, Oxford, 1991).

6. Schwartz, I. B. Small amplitude, long period outbreaks in seasonally driven epidemics. J. Math. Biol. 30, 473-491 (1992).

7. Zhou, G. et al. Association between climate variability and malaria epidemics in the East African highlands. Proc. Natl Acad. Sci. USA 101, 2375-2380 (2004).

8. Pascual, M. et al. Cholera dynamics and El Niño-Southern Oscillation. Science 289, 1766-1769 (2000).

9. Glass, R. I. et al. Endemic cholera in rural Bangladesh, 1966-1980. Am J. Epidemiol. 116, 959-970 (1982).

10. Koelle, K. \& Pascual, M. Disentangling extrinsic from intrinsic factors in disease dynamics: a nonlinear time series approach with an application to cholera. Am. Nat. 163, 901-913 (2004)

11. Lui, W. M., Hethcote, H. W. \& Levin, S. A. Dynamical behaviour of epidemiological models with nonlinear incidence rates. J. Math. Biol. 25, 359-380 (1987).

12. de Jong, M. C. M., Diekmann, O. \& Heesterbeek, H. in Epidemic Models: Their Structure and Relation to Data (ed. Mollison, D.) 84-94 (Cambridge Univ. Press, Cambridge, 1995).

13. Hastie, T. J. \& Tibshirani, R. J. Generalized Additive Models. Monographs on Statistics and Applied Probability (Chapman \& Hall, London, 1990).

14. Levine, M. M. \& Pierce, N. F. in Cholera (eds Barua, D. \& Greenough, W. B.) 285-327 (Plenum Medical, New York, 1992).

15. Clemens, J. D. et al. Biotype as determinant of natural immunising effect of cholera. Lancet 337, 883-884 (1991).

16. Pascual, M., Bouma, M. \& Dobson, A. P. Cholera and climate: revisiting the quantitative evidence. Microbes Infect. 4, 237-245 (2002)

17. Rodó, X. et al. ENSO and cholera: A nonstationary link related to climate change? Proc. Natl Acad. Sci. USA 99, 12901-12906 (2002).

18. Parthasarathy, B., Munot, A. A. \& Kothwale, D. R. Monthly and Seasonal Rainfall Series for all-India Homogeneous Regions and Meteorological Subdivisions: 1871-1994 (Research Report RR-065, Indian Institute of Tropical Meteorology, Pune, 1995).

19. Siddique, A. K. et al. Survival of classic cholera in Bangladesh. Lancet 337, 1125-1127 (1991)

20. Kawamura, R. A possible mechanism of the Asian summer monsoon-ENSO coupling. J. Met. Soc. Jpn $76-1027$ (1998)

21. Shukla, J. \& Fennessy, M. J. Simulation and Predictability of Monsoons 567-575 (Proc. Int. Conf. on Monsoon Variability and Prediction, Technical Report WCRP-84, World Climate Research Programme, Geneva, 1994).
22. Lau, N.-C. \& Nath, M. J. Impact of ENSO on the variability of the AsianAustralian monsoons as simulated in GCM experiments. J. Clim. 13, 4287-4309 (2000).

23. Lau, N.-C. \& Nath, M. J. Coupled GCM simulation of atmosphere-ocean variability associated with zonally asymmetric SST changes in the tropical Indian Ocean. J. Clim. 17, 245-265 (2004).

24. Webster, P. J. et al. in Meteorology at the Millennium. Int. Geophys. Series Vol. 83, 198-219 (Academic, New York, 2002)

25. Dixon, P. A., Milicich, M. J. \& Sugihara, G. Episodic fluctuations in larval supply. Science 283, 1528-1530 (1999).

26. Elsner, J. B. \& Tsonis, A. A. Singular Spectrum Analysis: A New Tool in Time Series Analysis (Plenum, New York, 1996).

27. Kaplan, D. \& Glass, L. Understanding Nonlinear Dynamics. Text in Applied Mathematics (eds Marsden, J. E. et al.) 342-346 (Springer, New York, 1995).

28. Waldor, M. K., Colwell, R. \& Mekalanos, J. J. The Vibrio cholerae 0139 serogroup antigen includes an O-antigen capsule and lipopolysaccharide virulence determinants. Proc. Natl Acad. Sci. USA 91, 11388-11392 (1994).

29. Glass, R. I. \& Black, R. E. in Cholera (eds Barua, D. \& Greenough, W. B.) 129-154 (Plenum Medical, New York, 1992).

30. Woodward, W. E. \& Mosley, W. H. The spectrum of cholera in rural Bangladesh. II. Comparison of El Tor Ogawa and Classical Inaba infection. Am J. Epidemiol. 96, 342-351 (1972)

Supplementary Information is linked to the online version of the paper at www.nature.com/nature.

Acknowledgements We thank B. Sack for discussions on cholera immunity, K. Streatfield for support with the cholera data, A. Dobson for comments on the manuscript, P. Webster and the Climate Forecast Applications Project at Georgia Tech for the river discharge data, and the Bangladesh Water Development Board, Dhaka, Bangladesh, for flood area data. M.P. acknowledges the joint support of the NSF-NIH (Ecology of Infectious Diseases) and NOAA (Oceans and Health), as well as funding from NOAA's Joint Program on Climate Variability and Human Health, with EPRI-NSF-NASA, under which the work was initiated. Further support was provided by the James S. McDonnell Foundation Centennial fellowship to M.P. and by ICREA and an AGAUR-DURSI grant to X.R.

Author Information Reprints and permissions information is available at npg.nature.com/reprintsandpermissions. The authors declare no competing financial interests. Correspondence and requests for materials should be addressed to M.P. (pascual@umich.edu). 